\title{
Single Atom Manipulation in a Microscopic Dipole Trap
}

\author{
Georges Reymond, Nicolas Schlosser and Philippe Grangier \\ Laboratoire Charles Fabry de l'Institut d'Optique, \\ UMR 8501 du CNRS, B.P. 147, 91403 Orsay Cedex - France \\ Tel : 33 (0)169358735, Fax: 33 (0)1 69358700 \\ E-mail : philippe.grangier@iota.u-psud.fr
}

\begin{abstract}
We describe the operation of a very small optical dipole trap that is designed to store and manipulate individual atoms. Due to the very small dipole trap volume, a "collisional blockade" mechanism locks the average number of trapped atoms on the value 0.5 over a large range of loading rates. We demonstrate experimentally the existence of this regime, and we describe also the "weak loading" and "strong loading" regimes outside the blockade range. In addition, we describe methods to measure the oscillation frequencies of a single atom in the trap with a high accuracy, and a "release and recapture" method designed for temperature measurements at the single atom level. The measured "single atom temperature" is $35 \mu K$, that is in the sub-Doppler regime. Finally, by exploiting the extremely high density observed in the strong loading regime, we present preliminary results for evaporative cooling, where typically 30 atoms at $200 \mu K$ are forced to evaporate into about 10 atoms at $15 \mu K$.
\end{abstract}

\section{Introduction}

There is currently a strong interest for the manipulation of individual neutral atoms in microscopic optical dipole traps $[1,3,4,5,6]$. Besides their fundamental interest, the manipulations of individual quantum objects may open the way to controlled engineering of the quantum state of small sets of trapped particles, in order to encode and process information at the quantum level $[7,8,9,10]$. We have demonstrated recently that it is possible to load and detect individual atoms in an optical dipole trap with a sub-micrometer size $[1,2]$. The dipole trap is initially loaded from a very low density magneto-optical trap (MOT), that cools the atoms and allows us to detect them easily from the induced fluorescence. Due to the extremely small trapping volume, only one atom can be loaded at a time, resulting in strongly sub-poissonnian statistics of the number $\mathrm{N}$ of atoms in the trap: one has either zero or one atom in the trap, with equal probabilities.

The "collisional blockade" mechanism [2] that creates this effect is due to an original combination of the well-studied behaviour of dipole traps in the presence of MOT light [11], and of new features that are specifically due to the very small volume of our trap. We will present a simple theoretical analysis, that allows us to identify three operating regimes ("weak loading", "blockade", and "strong loading"), as a function of the number of atoms that enter the dipole trap area. We will show that the novel "blockade" regime appears only if the trap is small enough. Then we will present experimental evidence for the observation of these three regimes in our dipole trap, that is characterized by a beam waist $w_{0}<1 \mu \mathrm{m}$. 


\section{Experiment}

The experimental set-up displayed on fig. 1 (see also [1, 2]) consists of a strongly focused dipole trap loaded from a magneto-optical trap (MOT). The MOT is loaded from an atomic beam, slowed down by chirped cooling using the usual "slower" and "repumper" laser diodes. The MOT density, that is proportional to the loading rate of the dipole trap, can be changed over many orders of magnitude. The highest values (about $10^{10} \mathrm{~cm}^{-3}$ ) are obtained with standard parameters, while the lowest ones (about $10^{5} \mathrm{~cm}^{-3}$ ) are obtained by turning off the slowing beams and the MOT magnetic field, and decreasing the oven temperature. The focusing objective consists

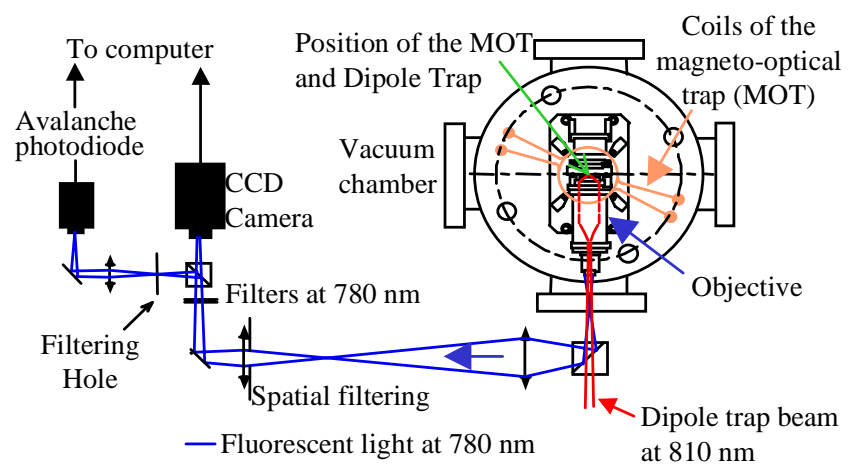

Figure 1: Main features of the experimental set-up. For simplicity, the slowing and MOT beams are not represented.

of two parts, which first focus the light inside the MOT and then re-collimates the beam for diagnostic purposes. The numerical aperture of 0.7 gives a diffraction-limited resolution of $0.7 \mu \mathrm{m}$ for the focusing part. The light beam injected in the objective comes from a c.w., frequency stabilized Titanium-sapphire laser, and it is brought to the set-up by using an optical fiber. A wide range of power and wavelength is thus available, and the dipole trap is usually operated in the "FORT" (Far Off Resonance) regime around $810 \mathrm{~nm}$ [13], with a few $\mathrm{mW}$ input power.

The fluorescence from the trapped atoms excited by the MOT light is collected by the same objective focusing down the beam (see fig. 1), and gives a magnified image of the trap on a CCD camera. Since the CCD camera has a slow response time, a photon-counting avalanche photodiode (APD) is used in parallel, and monitors only the light coming from the trap region : typically, the one-micron diameter dipole trap is imaged onto a fifty-micron pinhole. We note that all the photon counting is done at the MOT wavelength at $780 \mathrm{~nm}$ : the stray light elimination is obtained only by spatial filtering. On the other hand, the residual dipole trap stray light at $810 \mathrm{~nm}$ is eliminated by an interference filter. An atom in the dipole trap sees a large light shift, and therefore its fluorescence rate is weaker than the same atom in the MOT. This can easily be seen by suddenly turning off the dipole trap : before the atom leaves the observation area (a few cubic microns in size), its fluorescence rate shows a sharp increase that appears as a flash of light on the APD. The typical detection rate from a single atom in the dipole trap is about 5000 counts per second (cps), while it is about $20000 \mathrm{cps}$ in the MOT alone. 
(a)

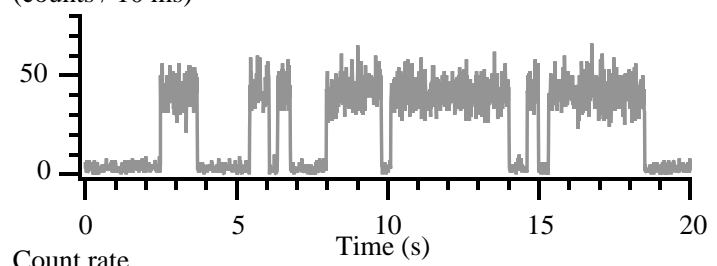

Count rate

(b)

(counts / $10 \mathrm{~ms}$ )

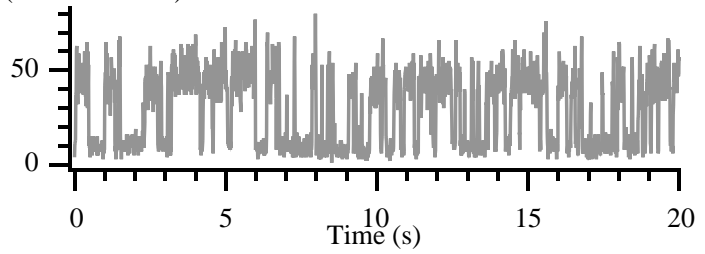

Figure 2: Comparison of the counting sequences in the weak loading (a) and blockade regime (b)

Fig. 2 shows typical time sequences of photons counted by the APD in $10 \mathrm{~ms}$ bins, when both the MOT and the dipole trap are continuously on. When the MOT density is small (weak loading regime, see below), the duration of the plateaus is essentially limited by the one-body decay due to collisions with the background gas (Fig. 2(a)). When the loading rate is increased, the probability to have one atom increases also, and then locks on the value 0.5. Simultaneously, the duration of the plateaus becomes shorter and shorter (Fig. 2(b)). The probability distribution of the atom number is easily obtained by looking at the histogram of the number of samples for a given fluorescence level, that is shown on figure 3(a). If the loading rate is increased further, the number of atoms starts fluctuating faster than the $10 \mathrm{~ms}$ counting time. One has thus to reduce this time, but the photon noise becomes a serious issue : only typically 5 photons are detected in $1 \mathrm{~ms}$, and it becomes difficult to separate the zero and oneatom peaks. Nevertheless, fig. 3(b) shows an histogram taken with a time bin of 2 $\mathrm{ms}$, in the intermediate regime that is close to the upper limit of the blockade regime. Fitting the histogram reveals that a two-atom contribution is appearing, though it is still not very large. Finally, one may go to high loading rates, and decrease the time bin to $0.5 \mathrm{~ms}$. The steps due to individual atoms are no more resolved, but the number of atoms can be determined by studying the statistical distribution of photons coming from the trapped atoms. Good agreement is also obtained in that case [2].

\section{Collisional blockade}

In order to interpret these results, we consider an optical dipole trap containing $N$ trapped atoms. The number $N$ will vary as a function of the loading rate $R$, the onebody decay $-\gamma N$ (essentially due to collisions with fast atoms from the background gas), and the two-body decay $-\beta^{\prime} N(N-1)$, that is due to a mixture of various inelastic 

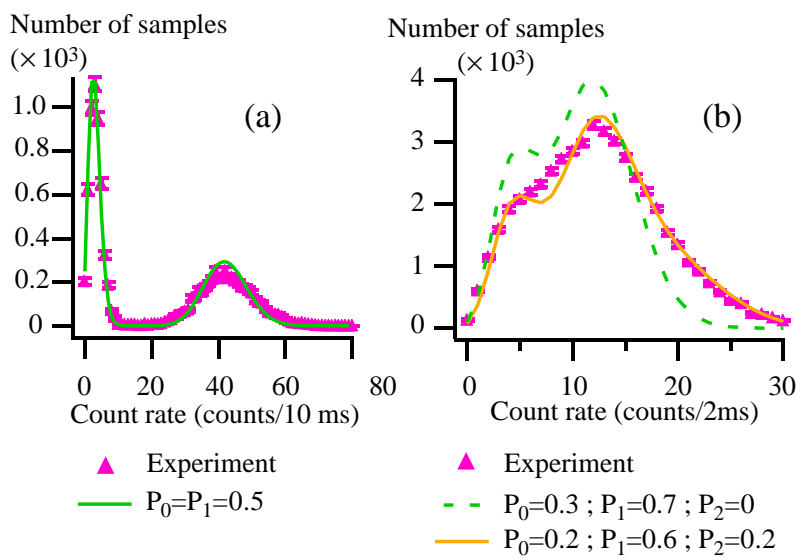

Figure 3: Histograms in the collisional blockade regime (a) and in the intermediate regime (b). Fits of this results give the probability $P_{n}$ of trapping $n$ atoms. The dash curve obtained with $P_{2}=0$ is unable to fit the experimental points in the intermediate regime.

collisional mechanisms, analysed in detail in [11]. The equation for $N$ is thus:

$$
\frac{d N}{d t}=R-\gamma N-\beta^{\prime} N(N-1)
$$

Since this equation is written for the atom number and not for the atomic density, the value of $\beta^{\prime}$ is inversely proportional to the volume of the trap [11] : the smallest the trap, the largest $\beta^{\prime}$. In the steady state, one can immediately identify two regimes:

- Weak loading : for small values of $R$, the effect of the collisional term is negligible, so that $\langle N\rangle \sim R / \gamma$.

- Strong loading : for large values of $R,\langle N\rangle$ becomes large, and the collisional term becomes important. The average number of atoms is then limited by the collisions, so that $\langle N\rangle \sim \sqrt{R / \beta^{\prime}}$. The cross-over between weak and strong loading is defined by a critical atom number $N_{c}=\gamma / \beta^{\prime}$, associated with a critical loading rate $R_{c}=\gamma^{2} / \beta^{\prime}$ above which the collisional term becomes dominant (see fig. 4). As long as $N_{c} \gg 1$, collisions play a role only when $\langle N\rangle$ is large, and one has simply a reduction in the slope of $\langle N\rangle$ vs $R$. But when $N_{c} \ll 1$, this reduction would appear even if $\langle N\rangle \ll 1$, what is not physically acceptable because at least two atoms are needed for a collision. In this case, collisions play a role as soon as there are two atoms in the dipole trap : this is the "collisional blockade" regime.

The simplest way to analyze this regime is to use a classical Monte-Carlo simulation, with random atom arrivals, including one-body and two-body decays. In the simulation, one has to choose whether a collision ejects both atoms from the trap, or only one; good agreement with the experiment is obtained by assuming that in the collision both atoms are ejected from the trap (otherwise, we would reach an average number of atoms $0.5<\langle N\rangle\langle 1$, contrary to the experiment). This is also consistent with what is expected from the underlying collisional mechanisms, even if one-atom losses have also been observed in other geometries [12]. 


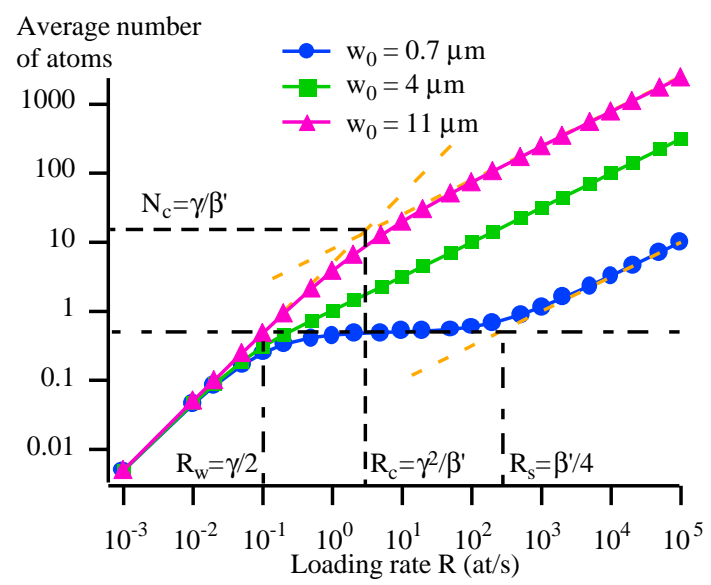

Figure 4: Average number of atoms in the trap as a function of the loading rate $R$ for different values of the waist. $w_{0}=11 \mu \mathrm{m}$ corresponds to a standard configuration. For $w_{0}=0.7 \mu \mathrm{m}$, the blockade regime is clearly seen.

In order to get quantitative predictions, one has to introduce numerical values for $\gamma$ and $\beta^{\prime}$. We will use $\gamma=0.2 s^{-1}$, that is consistent with our observations, and with the background pressure in our vacuum chamber (a few $10^{-10}$ Torr). The value of $\beta^{\prime}$ will be taken from ref. [11], that leads to $\beta^{\prime}=1000 \mathrm{~s}^{-1}$ for our trapping volume. This value is compatible with our observations. We note that $\beta^{\prime}$ reduces very quickly with the trap size (the volume of the trap is proportional to $w_{0}^{4}$ ), e.g. it drops to $\beta^{\prime}=0.016 \mathrm{~s}^{-1}$ for a more "standard" beam waist $w_{0}=11 \mu \mathrm{m}$. Fig. 4 shows in $\log$-log scale the number of trapped atoms as a function of the loading rate. This figure shows clearly that the blockade effect appears for a trap size that is typically smaller than $4 \mu \mathrm{m}$. In our operating conditions, it extends over about 3 orders of magnitude in loading rates, typically between $R=0.1$ and 100 atoms/sec. From the Monte-Carlo simulations [2], the atom statistics are sub-poissonian for any value of the loading rate, but this effect is particularly clear in the blockade regime where only $N=0$ and $N=1$ have a significant probability, that is close to $1 / 2$. The variance is then 0.25 , i.e. $0.5\langle N\rangle$ or "3 dB squeezing" $[1,2]$.

\section{Oscillation frequencies and temperature of the trapped atom}

Once one atom is loaded in the trap, the loading can be stopped simply by turning off the MOT. It is then important to characterize the dynamics of a single trapped atom, by measuring its temperature and oscillation frequencies in the dipole trap. For that purpose, a single atom is loaded in the trap, then the trapping laser beam is switched off for a short time interval, and then switch on again. This "kicks" the atom, that starts to oscillate in the trap. Then the trapping laser beam is switched off and on again. The probability of recapturing the atom after the second "off" pulse depends on the phase of the oscillation, and thus on the delay time $\Delta t$ between the two pulses. 


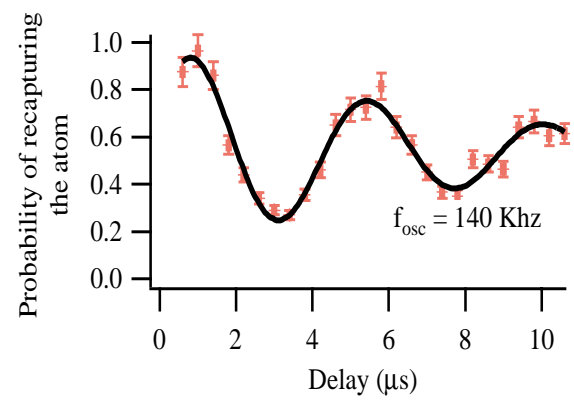

(a) $\Delta \mathrm{t}_{1}=2.5 \mu \mathrm{s}$

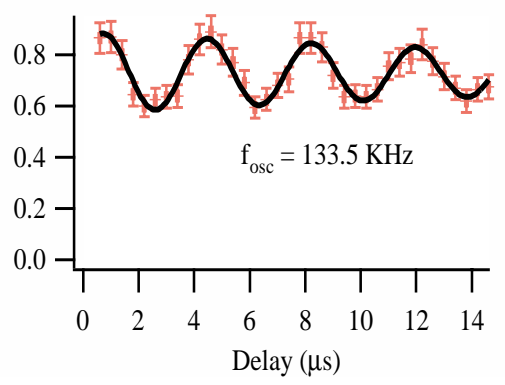

(b) $\Delta \mathrm{t}_{1}=1 \mu \mathrm{s}$

Figure 5: Oscillations of a single atom in the trap. In (b) the motion amplitude is smaller and is thus harmonic: there is almost no damping. Each point correspond to 200 successful events - i.e. with one atom at the beginning - for a given time delay between the two pulses.

Due to the symmetry of the motion, the probability of keeping the atom after the two pulses oscillates at twice the oscillation frequency.

The results are represented on Fig. (5) for two different durations $\Delta t_{1}$ of the kick pulse. In (a) the atom is strongly kicked, and the oscillations are quickly damped due to the anharmonicity of the trap potential. In (b), the kick is weaker, and thus the amplitude of the atom motion in the trap is also decreased. Therefore the potential probed by the atom remains mainly harmonic, and the oscillations stay longer. From our measurements we can infer a beam waist for the trapping beam of $w_{0}=0.9 \pm$ $0.05 \mu \mathrm{m}$. Taking a trapping beam power $P_{\text {trap }}=2 \mathrm{~mW}$ the typical parameters for our trap are $U_{0}=1.6 \mathrm{mK}, f_{x}=f_{y}=140 \mathrm{kHz}$, and $f_{z}=30 \mathrm{kHz}$.

The atom's temperature can be measured by reducing the previous time sequence to a single release-recapture pulse of adjustable duration $\Delta t$ : it is then a time of flight sequence but with a single atom. We infer the temperature by adjusting the experimental data with a Monte-Carlo simulation representing a thermal distribution at temperature T. The experimental points are plotted on Fig. 6 with two simulations, using either $\mathrm{T}=140 \mu \mathrm{K}$ (the Doppler limit for Rubidium), or $\mathrm{T}=35 \mu \mathrm{K}$. It appears clearly that the temperature is below the Doppler limit and is around $35 \mu \mathrm{K}$.

Finally, by going to the strong loading regime, more than 30 atoms can be loaded in the trap, at a temperature of $190 \mu K$, measured by the method described above. Then forced evaporative cooling can be induced by decreasing the laser power (and therefore the trap's depth) from $10 \mathrm{~mW}$ down to $0.9 \mathrm{~mW}$. The number of atoms, as well as the temperature, clearly decrease when the power drops below $5 \mathrm{~mW}$, and about 10 atoms are still there for $1 \mathrm{~mW}$ trapping power. The release-and-recapture results are displayed on Fig. 7, showing clearly a decrease in temperature, that is accompanied by an increase in phase-space density by a factor 20 . This is a clear evidence that evaporative cooling is taking place. Though condensation has not been observed yet, this observation suggests stimulating perspectives towards Bose-Einstein "nanocondensates", containing a few atoms in the ground state of the trap. 


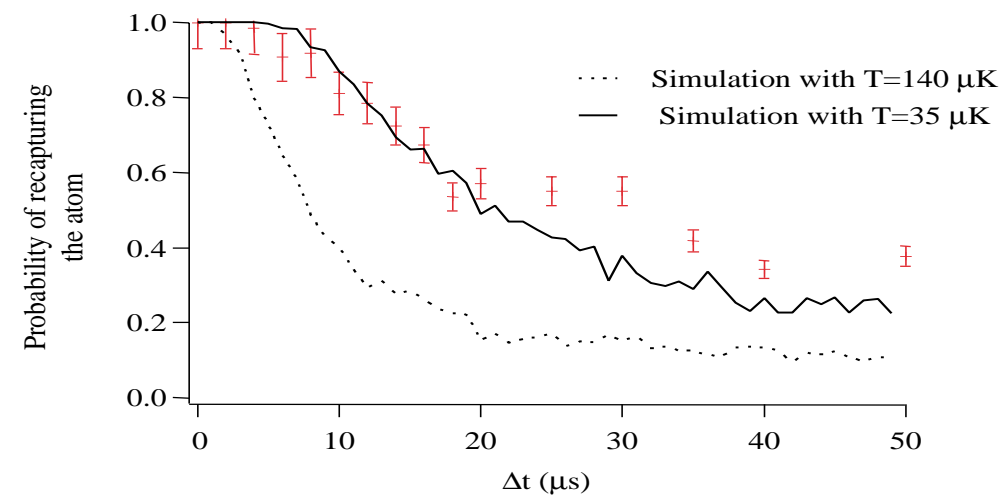

Figure 6: Release and recapture curves with a single atom: the experimental data fits with a temperature of $35 \mu \mathrm{K}$, well below the Doppler limit of $140 \mu \mathrm{K}$.

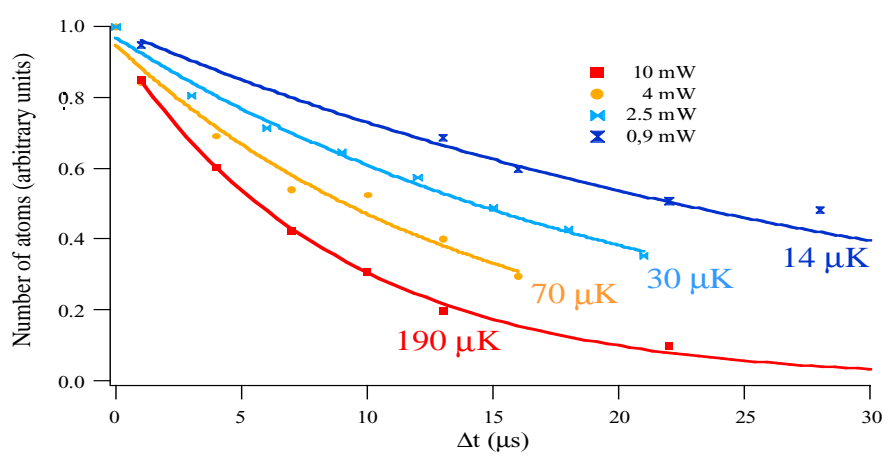

Figure 7: Release and recapture curves with many atoms, after forced evaporative cooling starting with a trapping power of $10 \mathrm{~mW}$. The number of recaptured atoms after a given time (normalized to the number of atoms at time zero) gives the temperature, that is clearly decreasing as more and more atoms evaporate. 


\section{Conclusion}

We have thus demonstrated a "collisional blockade" mechanism that locks the atom number or either zero or one in a very small dipole trap. This effect can be analyzed using the standard collisional mechanisms already known in combined MOT and dipole traps, provided that the trap volume is so small that collisions become the dominant loss mechanism as soon as there are two atoms in the trap. We have also shown that it is nevertheless possible to "bypass" the blockade regime, opening the possibility to have a large number of cold atoms in an extremely confined volume.

In order to progress towards quantum gates and atom-atom entanglement, we have sent another trapping beam at a small angle in the same optics, in order to trap two atoms at a controlled distance, that can be easily adjusted in the range 1-10 $\mu \mathrm{m}$ [14]. Then it may be possible to implement a Rydberg state scheme inspired from a proposal by the Innsbruck group [15].

We have also proposed a novel scheme for "conditional quantum logic", where an arbitrary unitary operation (i.e. a CNOT gate, a Bell measurement...) is realized on a pair of trapped atom (the two qubits), upon the detection of a single scattered photon (the trigger) [16]. This scheme is "conditional" in the sense that the gate does not always succeed, but one knows when it does. Conditional gates are for instance the basis of the proposed scheme for linear quantum computing [17], and in order to make such gates scalable, one should use some kind of quantum teleportation scheme. It this thus clear that many possibilities are open towards quantum information processing using trapped neutral atoms.

\section{References}

[1] N. Schlosser, G. Reymond, I. Protsenko and P. Grangier, Nature 411, 1024 (2001).

[2] N. Schlosser, G. Reymond and P. Grangier, Phys. Rev. Lett. 89, 023005 (2002).

[3] S. Kuhr et al, Science 293, 278-280 (2001).

[4] M. Greiner et al, Nature 415, 39-44 (2002).

[5] A.C. Doherty, et al, Phys. Rev. A 63, 013401 (2001)

[6] P.W.H. Pinkse, T. Fischer, P. Maunz, G. Rempe, Nature 404, 365-8 (2000).

[7] C.A. Sackett et al, Nature 404, 256 (2000).

[8] A. Rauschenbeutel et al, Phys. Rev. Lett. 83, 5166 (1999).

[9] T. Calarco et al, J. Mod. Opt. 47, 2137 (2000).

[10] G.K. Brennen, I.H. Deutsch, P.S. Jessen, Phys. Rev. A 61, 062309 (2000).

[11] S. J. M. Kuppens et al, Phys. Rev. A 62, 013416 (2000).

[12] B. Ueberholz et al J. Phys. B 33, L135-L142 (2000).

[13] J.D. Miller, R.A. Cline, D.J. Heinzen, Phys. Rev. A 47, R4567 (1993)

[14] I.E. Protsenko, G. Reymond, N. Schlosser and P. Grangier, Phys. Rev. A, 65, 052301 (2002).

[15] D. Jaksch et al, Phys. Rev. Lett. 85, pp.2208-2211, (2000).

[16] I.E. Protsenko, G. Reymond, N. Schlosser and P. Grangier, "Conditional quantum logic", arXiv:quant-ph/0206007.

[17] E. Knill, R. Laflamme and G.J. Milburn, Nature 409, 46 (2001). 